\title{
The impact of Stul digestion in situ on FISH to human chromosomes with satellite DNA probes
}

\author{
M Nieddu, G Pichiri, V Melis and R Mezzanotte \\ Dipartimento di Scienze Applicate ai Biosistemi, Sezione di Biologia e Genetica, Cittadella Universitaria, 09042 Monserrato (CA), Italy
}

\begin{abstract}
Human metaphase chromosomes were digested with Stul and subsequently hybridized in situ using chromosome 9 alphoid DNA and classical satellite III DNA as probes. The data obtained suggest that it is not possible to establish a general rule regarding the cytological effects induced by
\end{abstract}

restriction enzymes in particular chromosome regions and that a number of factors, such as DNA sequences, DNAprotein interaction and enzyme structure, play a role in determining such effects.

Heredity (2003) 90, 298-301. doi:10.1038/sj.hdy.6800238

Keywords: endonucleases; DNA; chromosomes; satellite DNA; FISH

\section{Introduction}

Centromeric/paracentromeric chromatin contains two main types of highly repetitive DNAs in human chromosomes: (i) alphoid and (ii) classical satellite sequences, the former being made up of a basic $171 \mathrm{bp}$ subunit (see Tyler-Smith and Willard, 1993), and the latter consisting of three major types of satellite DNAs, which can be separated by means of ion-cesium sulphate gradients (Frommer et al, 1982) and called I, II and III. In turn, satellites II and III are closely related and composed of a mixture of different repeated sequences, although containing simple sequences, respectively called S2 and S3, as major components (Meyne et al, 1994). In this connection, it is noteworthy that the simple S2 and S3 sequences appear to derive from the $5 \mathrm{bp}$ sequence ATTCC (Grady et al, 1992).

Restriction enzymes (REs) have been used in molecular cytogenetics in an attempt to study the localization and organization of repetitive DNA sequences in eukaryote chromosomes (Mezzanotte et al, 1983a; Miller et al, 1983). In this connection, Nieddu et al (1999) have recently shown that $A l u \mathrm{I}$ and TaqI act on human metaphase chromosomes by cleaving but removing only limited amounts of some alphoid DNAs. As a consequence, chromatin reorganization is induced which, in turn, produces an enhancement in the fluorescent in situ hybridization (FISH) signal using specific alphoid DNAs as probes.

We attempt here to confirm whether or not the action of REs in situ alters the organization of specific centromeric/paracentromeric regions so as to affect the results of subsequent FISH. To this end, we employed StuI, an enzyme known for its ability to cleave the basic $171 \mathrm{bp}$ subunit of human alphoid DNAs (Tagarro et al,

Correspondence: $R$ Mezzanotte, Dipartimento di Scienze Applicate ai Biosistemi, Sezione di Biologia e Genetica, Università di Cagliari, Cittadella Universitaria, 09042 Monserrato (CA), Italy.

E-mail: mezzanotte@unica.it

Received 24 April 2002; accepted 26 September 2002
1993). FISH experiments were thus carried out using alphoid DNA and a specific S3 sequence localized in the centromeric/paracentromeric chromosome 9 region as a probe, in both StuI-treated and -untreated human metaphase chromosomes. The results obtained indicate that the RE-structure, which has been the subject of few biochemical studies to date, may play an important role in determining the cytological effects relative to in situ digestion.

\section{Materials and methods}

\section{Cytological experiments}

Human cytological preparations were obtained from peripheral blood lymphocytes of healthy donors, according to standard procedures. Preparations were air-dried for $24 \mathrm{~h}$ and subsequently digested in situ with $30 \mathrm{U}$ of StuI (Gibco BRL, Life Technologies) for $16-18 \mathrm{~h}$ at $37^{\circ} \mathrm{C}$ according to Mezzanotte et al (1983a). FISH was carried out on untreated preparations as well as on preparations previously treated with StuI. The probes used in the present study were: (1) human alphoid DNA (pMR9A), localized at the centromere of chromosome 9 (furnished by M Rocchi, Genetics Institute, University of Bari, Italy) and labelled using biotin-16-dUTP, according to the nick-translation procedure described by the supplier (Roche) and (2) a single-stranded oligonucleotide, obtained by automated synthesis (Amersham Phaarmacia Biotech), designated S3, and belonging to human classical satellite DNA III, whose sequence is $5^{\prime}$-TCC ACT CGG GTT GAT T-3' (16-mer). As stated above (see the Introduction), simple repeated sequences of S2 and S3 share the $5 \mathrm{bp}$ ATTCC sequence (Grady et al, 1992); therefore, to avoid cross-hybridization between S2 and S3, we prepared the above-described oligonucleotide, specific for S3 DNA, without the ATTCC consensus sequence, according to Tagarro et al (1994). Labelling was effected at the $5^{\prime}$ end with biotin by Amersham Pharmacia Biotech. FISH was carried out according to Nieddu et al (1999). 


\section{Biochemical experiments}

Genomic DNA was isolated from peripheral blood lymphocytes according to Maniatis et al (1982). DNA was also obtained after StuI digestion in situ from the material solubilized (so-DNA) and from the material retained on the slides (re-DNA), according to Nieddu et al (1999). Agarose-gel electrophoresis was carried out for $2 \mathrm{~h}$ at $100 \mathrm{~V}$, and DNA samples were subsequently transferred onto Hybond $\mathrm{N}+$ filters (Amersham). The nonradioactive ECL method (Enhanced chemioluminescence Kit, Amersham) was used to hybridize alphoid probe pMR9A, while the S3-specific oligonucleotide was employed using a $3^{\prime}$ oligo labelling and detection system (Amersham).

\section{Results and discussion}

Filter hybridization carried out using chromosome 9 alphoid DNA on genomic DNA digested with StuI produces an electrophoretic pattern showing the majority of such highly repetitive DNA in the so-DNA fraction and revealing hybridization bands identical to those observed in genomic DNA, the size of DNA fragments ranging from about $3 \mathrm{~kb}$ to $350 \mathrm{bp}$ (Figure 1a). On the contrary, Southern blotting using a consensus sequence belonging to S3 DNA (Tagarro et al, 1994) as a probe shows that this highly repetitive DNA is not cleaved by StuI and is almost entirely retained on the slides, since no appreciable amounts are found in the so-DNA fraction (Figure 1b).

FISH experiments show that fluorescent signal intensity dramatically decreases in chromosomes predigested with StuI as compared to control, untreated chromosomes subsequently hybridized using chromosome 9 alphoid DNA as a probe (Figure $2 a$ and b). On the contrary, clearcut enhancement in fluorescent signal is observed in the same type of experiment when chromo- somes pretreated with StuI are hybridized using S3 DNA (Figure $3 a$ and $b$ ).

StuI cleaves human alphoid DNAs into $171 \mathrm{bp}$ fragments, the relative restriction site being present within the monomeric repeating subunit sequence (Manuelidis, 1978). It is thus not surprising that a large number of such sequences are solubilized during StuI in situ digestion as a consequence of specific DNA removal and loss, and that the fluorescent intensity of FISH signal decreases when in situ hybridization is carried out using alphoid DNA as a probe. This fact apparently contrasts with the data reported by Nieddu et al (1999); these authors, in fact, report that (i) the same alphoid DNA (chromosome 9) is extensively cleaved by both AluI and TaqI, but (ii) the fluorescent signal found in FISH experiments carried out after digestion in situ with the above-cited enzymes increases. Since the cleavage efficiency of either AluI or TaqI on this alphoid DNA is similar to that shown by StuI, we explain this discrepancy by postulating that different structures of different REs might facilitate/inhibit enzyme attack on nucleoproteic centromere organization. This hypothesis is confirmed by the following observation: digestion with StuI, whose base sequence target is $5^{\prime}$-AGG $\downarrow$ CCT-3', is known to induce unstained gaps in human centromeres with either Giemsa or propidium iodide, this fact being interpreted as a direct consequence of extensive digestion of alphoid human DNAs (Tagarro et al, 1993). On the contrary, digestion of human chromosomes with HaeIII, whose base sequence target is $5^{\prime}-\mathrm{GG} \downarrow \mathrm{CC}-3^{\prime}$, produces a G-plus C-banding pattern (Mezzanotte et al, 1983b) but no evident unstained centromeric gaps. This is noteworthy since (a) the base sequence target of the four-base cutter HaeIII is the central tetranucleotide (5'-GG $\downarrow$ CC-3'), belonging to the base sequence target of the six-base cutter StuI, and (b) four-base cutters should be more efficient than six-base cutters, due to cleavage frequency, higher in the former than in the latter (Modrich and
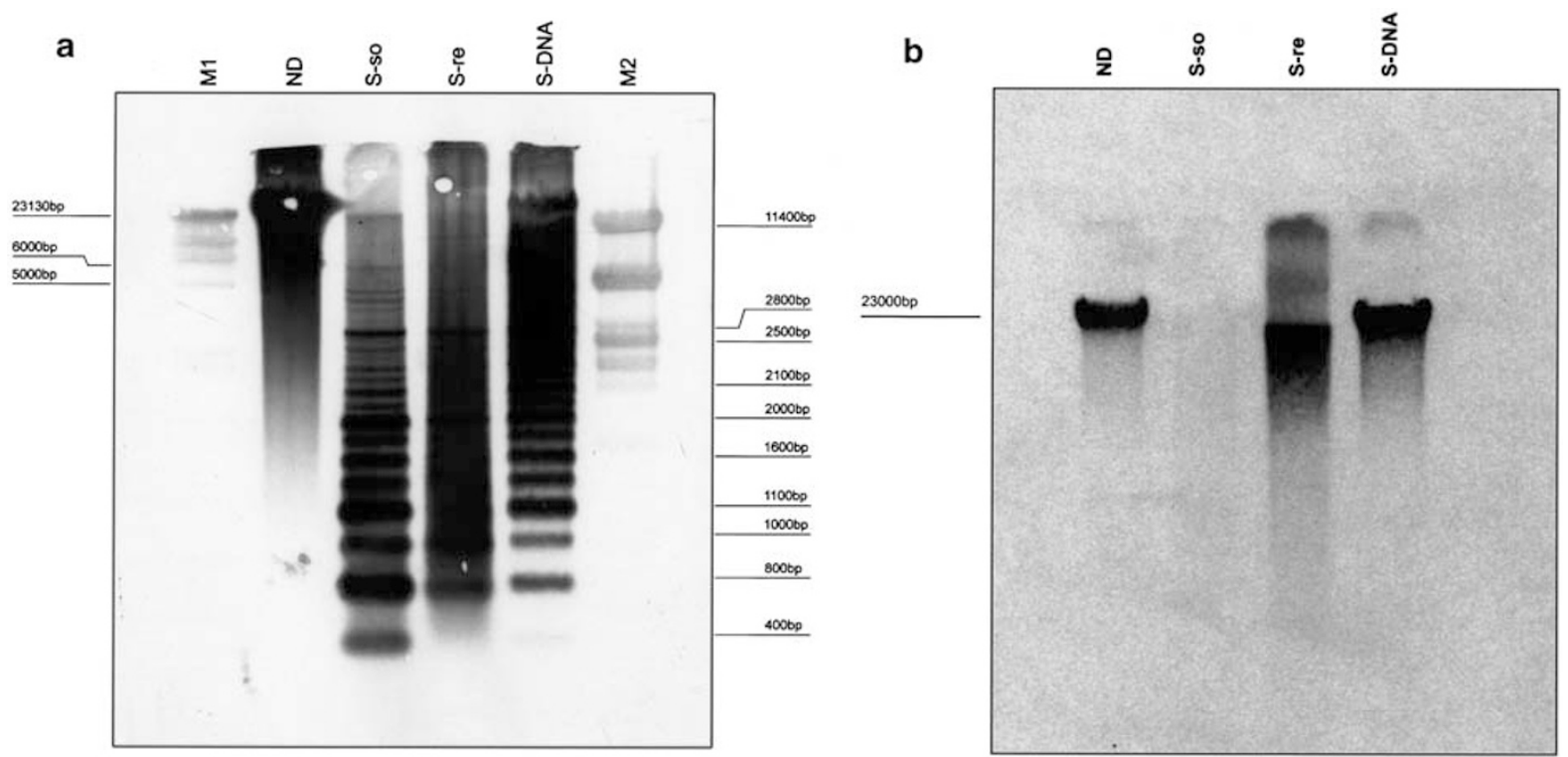

Figure 1 Filter hybridization obtained using chromosome 9 alphoid DNA (a) and classical satellite III (b) as probes. In (a): M1 and M2 are phage $\lambda$ DNA cleaved, respectively, with HindIII and PstI as markers. In both (a) and (b), the hybridization patterns are shown in undigested genomic human DNA (ND), the DNA solubilized (so-DNA) and the DNA retained on the slide (re-DNA) after StuI digestion in situ and naked genomic DNA digested with StuI (S-DNA). 

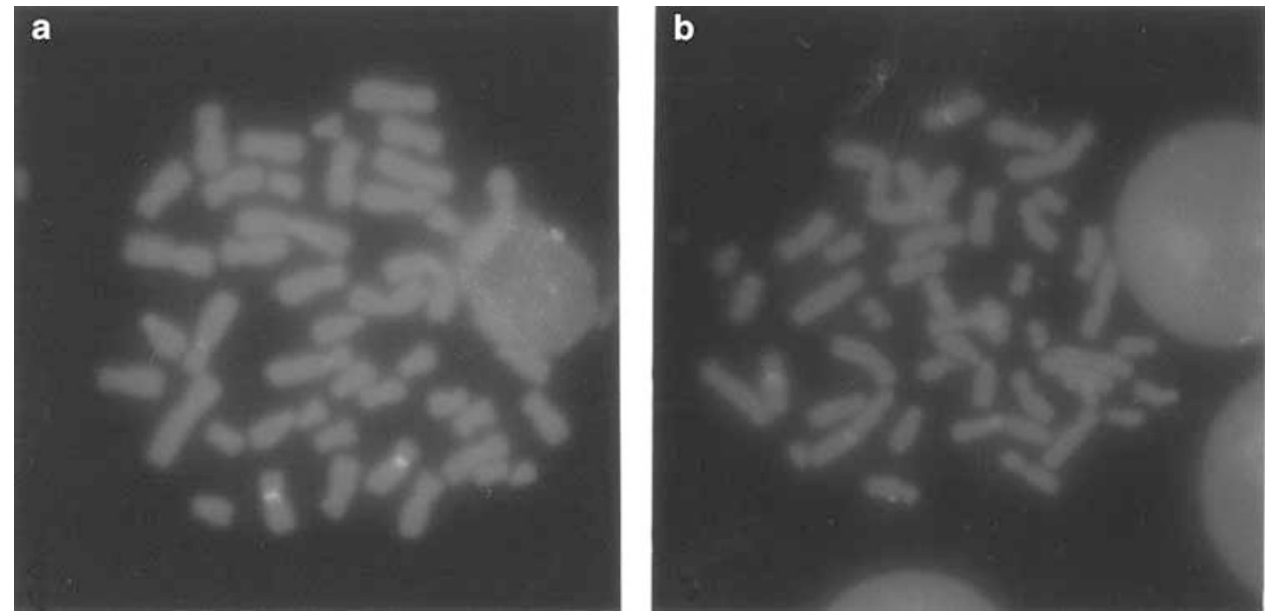

Figure 2 In situ hybridization carried out using chromosome 9 alphoid DNA as a probe in (a) standard, untreated chromosomes and (b) chromosomes pretreated with StuI. Note the decreased intensity of the hybridization signal in (b) as opposed to (a).
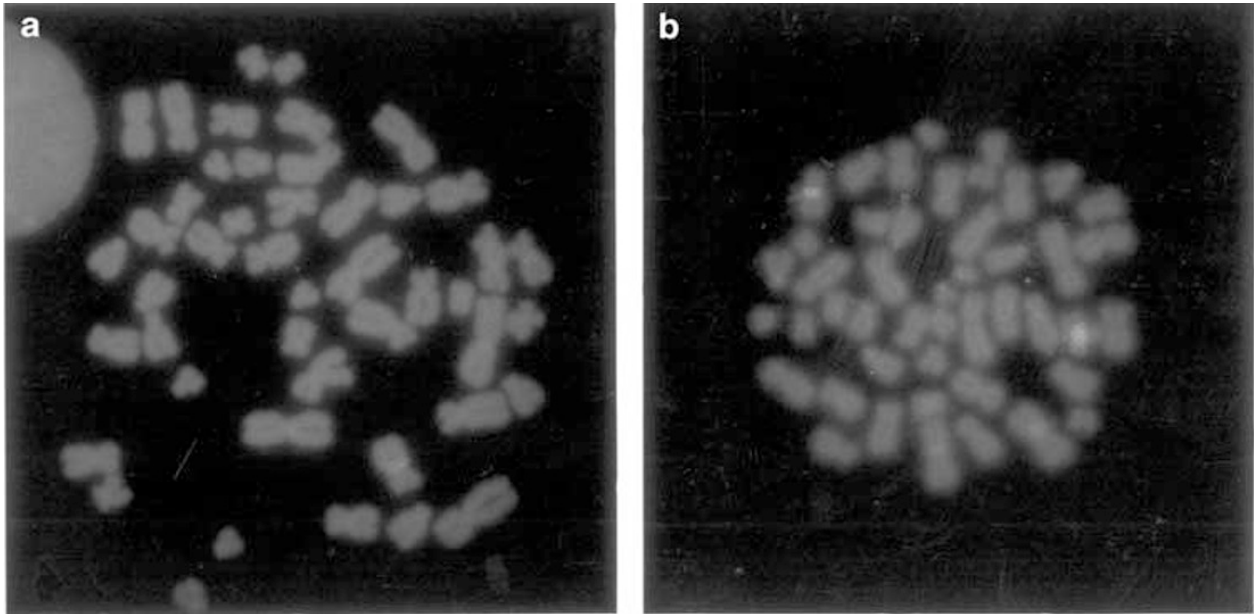

Figure 3 In situ hybridization carried out using classical satellite III DNA as a probe in (a) standard, untreated chromosomes and (b) chromosomes, pretreated with StuI. Note the enhanced intensity of hybridization signal in (b) as compared to (a).

Roberts, 1982). Therefore, the different longitudinal banding observed in human chromosomes when digestion is carried out with StuI as compared to HaeIII would further suggest that enzyme structure, intended as the spatial configuration of the enzymatic proteic component, is a critical factor in determining different interactions among different REs and the organization of specific chromosome regions. On the other hand, the increase in fluorescent signal in human chromosomes pretreated with $S t u \mathrm{I}$ and subsequently hybridized using a sequence belonging to the highly repeated S3 structure, agrees with the data reported by Nieddu et al (1999), but contrasts with previous data by Fernández et al (1997). Our biochemical results reveal that the sequence we used as a probe for S3 DNA is not cleaved by StuI. Similarly, Moyzis et al (1987) maintain that no AluI sites are present within the base sequence (D9Z1) subsequently employed by Fernández et al (1997). Therefore, since both probes are localized in the 9 q12 paracentromeric area, it is noteworthy that, contrary to the increase in FISH signal found in our experiments, Fernández et al (1997) report no significant difference in the fluorescent signal observed in AluI-treated as compared to-untreated chromosomes. Again, we hypothesize that the action of REs depends strictly on their structure, possibly varying in different restriction enzymes, and that such action may be inhibited or favoured in particular chromatin domains, such as those containing highly repetitive DNAs. According to Nieddu et al (1999), our data imply that StuI cleaves DNA sequences adjacent to, although different from, the sequence belonging to S3 used as a probe. The result would be the reorganization of paracentromeric heterochromatin which, in turn, would enhance FISH efficiency because of an increased possibility of interaction between chromosomal DNA and DNA probe during in situ hybridization.

The data here reported indicate that it is not possible to draw general conclusions regarding the cytological effects induced by in situ RE-treatment. As already postulated (Gosálvez et al, 1997), in fact, such effects seem to depend on a number of factors, such as 
DNA sequence, DNA-protein interaction in specific chromosome regions and RE-structure, which may vary from case to case.

\section{Acknowledgements}

The authors thank Mary Ann Groeneweg for checking the English of the manuscript.

\section{References}

Fernández JL, Valverde D, Goyanes V, Buno I, Gosálvez J (1997). AluI in situ digestion of human alphoid and classical satellite DNA regions. High resolution digital image analysis of FISH signals from condensed and extended chromatin. Cytogenet Cell Genet 76: 94-100.

Frommer M, Prosser J, Tkachuk D, Vincent PC (1982). Simple repeated sequences in human satellite DNA. Nucleic Acids Res 10: 547-563.

Gosálvez J, López-Fernández C, Goyanes V, Mezzanotte R (1997). Chromosome differentiation using nucleases: an overview. In: Henriques-Gil N, Parker JS, Puertas MJ (eds). Chromosomes Today, Vol. 12. pp 23-49.

Grady DL, Ratliff RL, Robinson DL, McCanlies EC, Meyne J, Moyzis RK (1992). Highly repetitive DNA sequences are present at human centromeres. Proc Natl Acad Sci USA 89: 1695-1699.

Manuelidis L (1978). Complex and simple sequences in human repeated DNAs. Chromosoma 66: 1-21.

Meyne J, Goodwin EH, Moyzis RK (1994). Chromosome localization and orientation of the simple sequence repeat of human satellite I DNA. Chromosoma 103: 99-103.

Mezzanotte R, Ferrucci L, Vanni R, Bianchi U (1983a). Selective digestion of human metaphase chromosomes by $A l u I$ restriction endonuclease. J Histochem Cytochem 31: 553-556.

Mezzanotte R, Bianchi U, Vanni R, Ferrucci L (1983b). Chromatin organization and restriction endonuclease activity in human metaphase chromosomes. Cytogenet Cell Genet 36: 562-566.

Miller DA, Choi YC, Miller OJ (1983). Chromosome localization of highly repetitive human DNAs and amplified ribosomal DNA with restriction enzymes. Science 219: 395-397.

Modrich P, Roberts RJ (1982). Type II restriction and modification enzymes. In: Linn SM, Roberts RJ (eds). Nucleases. Cold Spring Harbor Laboratory: Cold Spring Harbor. pp 109-154.

Moyzis RK, Albright KL, Bartholdi MF, Cram LS, Deaven LL, Hildebraand CE et al (1987). Human chromosome-specific repetitive DNA sequences: novel markers for genetic analysis. Chromosoma 95: 375-386

Nieddu M, Rossino R, Pichiri G, Rocchi M, Setzu MD, Mezzanotte R (1999). The efficiency of in situ hybridization in human chromosomes with alphoid DNAs is enhanced by previous digestion with AluI and TaqI. Chromosome Res 7: 593-602.

Tagarro I, Fernández-Peralta AM, Gonzáles-Aguilera JJ (1993). Digestion of centromeric DNA from each human metaphase chromosome by the 6-bp restriction enzyme StuI. Histochemistry 99: 453-456.

Tagarro I, Wiegant J, Raap AK, Gonzáles-Aguilera JJ, Fernández-Peralta AM (1994). Assignment of human satellite I DNA as revealed by fluorescent in situ hybridization with oligonucleotides. Hum Genet 93: 125-128.

Tyler-Smith C, Willard HF (1993). Mammalian chromosome structure. Curr Opin Genet and Dev 3: 390-397. 